\title{
Concerns Regarding Food Biotechnology: An Ongoing Debate
}

\section{Fatme Al Anouti}

Natural Science and Public Health Department, Zayed University, Abu Dhabi, UAE

"Corresponding author: Fatme Al Anouti, Natural Science and Public Health Department, Zayed University, Abu Dhabi, UAE, Tel: 971-503-019-807; E-mail: Fatme.AlAnouti@zu.ac.ae

Received date: Feb 21, 2014, Accepted date: May 01, 2014, Publication date: May 05, 2014

Copyright: ( 2014 Al Anouti F. This is an open-access article distributed under the terms of the Creative Commons Attribution License, which permits unrestricted use, distribution, and reproduction in any medium, provided the original author and source are credited.

\begin{abstract}
The development of agricultural biotechnology has led to the production of numerous types of genetically modified crops with improved traits. The prevalence of genetically modified food is increasing globally. The Rapid progress in the development of genetically modified food has significantly however increased public concern about food safety in recent years. The appearance of such foods in the global market has lately resulted in debate and scientific discussion. This debate is likely to continue, probably in the broader context of other uses of biotechnology and their consequences for human societies. To date, regulations and policies which mandate the labeling of genetically modified food are strictly adhered to in many countries to allow consumers both right for knowledge and freedom of choice. Nonetheless, several countries have not yet implemented policies that would oblige food producers to label modified food products. Main concerns about the safety of genetically modified food include allergenicity, emergence of antibiotic resistance and out-crossing of wild type crop. On the other hand, genetically modified crops can successfully reduce maturation time and improve quality by increasing tolerance to stress and some plant diseases. In fact many debate and believe that genetically modified food is the solution of world hunger because it increases food security every day and guarantees adequate nutrition. The debate would likely continue in the future but until clear evidence is provided, all concerns can be considered relative and dealt with transparency and objectivity to reveal both the good and evil side of biotechnology.
\end{abstract}

\section{Introduction}

Today, modern techniques of biotechnology make it possible to introduce one or more genes; transgenes; into a variety of organisms. Food biotechnology refers to the technology of manipulating or modifying the DNA of an organism or crop in order to improve the quality and safety of foods [1]. Biotechnology employs the tools of genetic engineering to improve plants, animals, and microorganisms for food production. The development of agricultural biotechnology has led to the production of numerous types of genetically modified crops with improved traits. The prevalence of genetically modified food (GMF) is increasing globally. In the year 2001, the global area of commercially grown transgenic plants was 52.6 million hectares. The main GM crops were soybean occupying $63 \%$ of global transgenic area, followed by corn, cotton and canola which occupied respectively $19 \%, 13 \%$ and $5 \%$ of global transgenic cultivation area [2-4]. The first approval of a genetically engineered plant for human consumption was given in the United States in 1992 when the Flavr Savr tomato from Calgene received approval from United States Department of Agriculture and Animal and the Plant Health Inspection Service and food and drug administration. Since then, approximately 27 different food products have also been approved in other countries including the European Union [5]. Gene revolution in crop improvement in this era is a relatively complex process which involves the utilization of new genes derived from different kinds of living organisms in order to add new improved characteristics to the manipulated plant crop [4]. This technology is known as genetic engineering or genetic modification and the crops which are modified are referred to as genetically modified organisms (GMOs) or just GM crops [4]. The World Health Organization (WHO) defines the process of producing genetically modified organisms as "the splicing of a gene of interest from a donor organism and pasting it into a recipient to express the desired characteristic" [3]. All living organisms contain genes which code for information that determines the characteristics. The genetic material of the GM Food is altered in a way that does not happen naturally by multiplication or natural recombination [3]. In genetic modification, the intended gene is incorporated into the genome of a crop using a vector which contains several other genes and DNA sequences, including a promoter, transcription terminators, antibiotic resistance marker genes and other reporter genes [6]. A gene construct consists typically of three fundamental elements. The promoter functions as the on/off switch for the activation of the inserted modified gene in the recipient plant. The inserted modified gene or (structural gene) encodes a specifically selected trait. The terminator operates as a stop signal for transcribing the inserted altered gene [7].

Although in GMF safety the role of the intended gene is very important, the potential effects of these other genes need also to be assessed otherwise the insertion of the vector could contribute to the overall effect [6]. Numerous methods have been developed that are used to introduce and integrate foreign DNA into plant cells, leading to transformed plant phenotypes [5]. There are methods used in the transformation of approved agricultural crops such as biological vectors, physical or chemical methods [5]. It is very essential to ensure that any transformation system should allow for stable integration into the host genome without structural alterations of the foreign DNA and maintain the stability of the new phenotype over several generations [5]. Recombinant DNA transformation methods usually use modified plasmids derived from the bacterium known as of Agrobacterium tumefaciens [5]. This is a binary vector system which harbors the gene to be transferred and other genes encoding the necessary functions for transformation to occur [5]. This method ensures that a defined region of the presented DNA is precisely transferred to the new host genome. Other transformation methods are based on physical and chemical 
principles [5]. DNA fragments are bound to the surface of minute metal particles and shot into plant cells using specially developed device. The chemical method utilizes polyethylene glycol (PEG) or calcium chloride to facilitate the entry of foreign DNA through the plant cell wall [5].

\section{Public health and safety concerns}

The main concern in GMF safety is related to the direct effect of the expression of the main intended gene after insertion into the plant genome via a gene construct. An additional concern is that this may also cause significant, indirect and unintended effects on the expression and functionality of the plant's own genes, the number of copies of the construct inserted and their location in the plant genome are of a particular importance in this respect, with the possibility that many unexpected changes may occur [5]. There is a growing concern regarding the introduction of foreign genes into food plants because of unexpected or negative impact on human health (Table 1). A study that examined the effects of consumption of GM potatoes in rats showed appreciable differences in the intestines of these rats as compared to rats fed unmodified potatoes [5]. One of the major health concerns with GMF is its potential to increase allergies in the human population through the food chain. The possibility of fatal anaphylaxis in sensitized individuals after their unwitting exposure to allergenic proteins in unlabeled GM foodstuffs is a real danger. There are several examples for the demonstration of allergenicity of the Brazilian nut $2 \mathrm{~S}$ seed storage protein in transgenic soybean. It is clear that, if and when it is known that the protein gene is derived from a source with a history of allergenicity, there is a reasonable certainty that the GM crop will be allergenic [5]. Unfortunately, the reverse is not true: the use of a gene from something that is not allergenic will not guarantee that the GM crop will not possess allergenicity [5]. Another risk factor is the potential emergence of antibiotic resistance. This may develop because of the unintentional or accidental insertion of an antibiotic resistance gene (which is used as a marker gene to select for GMF from unmodified food during production) into the consumers' (and even the microbial flora) genetic make-up. Hence, diseases that once could be treated by existing antibiotics may become resistant to treatment [1]. The third concern to human health is the out-crossing that is defined by the World Health Organization as" the movement of genes from GM plants into conventional crops or related species in the wild, as well as the mixing of crops derived from conventional seeds with those grown using GM crops" [3]. This is why some countries implement strategies to minimize out-crossing by counting an obvious separation of the GM crops fields from conventional crops fields [3]. Growing GM crops on a large scale creates a potential environmental risk due to imbalance between the wildlife and the environment [3]. A study conducted by USDA, the US. Environmental Protection Agency (EPA) and other non-government research groups had shown that pollen from BT-corn (corn which produces a protein toxin from the bacterium Bacillus thuringiensis (BT) that kills certain insects) caused high mortality rates in monarch butterfly caterpillars. Concerns are rising regarding the emergence of insects with resistance to BT or other crop that have been genetically modified to create their own pesticides [3].

\begin{tabular}{|l|l|}
\hline Safety Concerns & Benefits \\
\hline Allergenicity & Less Pesticide Use, Better Pest Management and More Acreage Use \\
\hline Emergence of Antibiotic Resistance & More Nutritional Value and Reduced Prices \\
\hline Out-Crossing of Wild Type Crops & Food Security \\
\hline
\end{tabular}

Table 1: Safety Concerns versus Benefits regarding the Use of GMF.

\section{Future challenges and prospects in safety assessment:}

Crop biotechnology has been the single most rapidly adopted agricultural technology in history. Most biotechnology companies and biotechnology industries strive to find the right combination of agronomic and gene enhanced qualities to achieve high yielding, and high quality crops [8]. Farmers have found some uniform benefits to the use of GMO varieties because these crops require less pesticide use, allow farmers to plant more acreage with less labor and equipment, better pest management, and more economic profit regardless of the high price of GMO seeds [8]. Those benefits are not exclusive for producers but are also relevant to consumers in terms of nutritional value, lower price and durability (Table 1 ). The primary intention for developing plants based on GM organisms was to improve and increase the level of crop protection through resistance to insects and viruses and tolerance to herbicides. Insect resistance is attained by incorporating into the food plant the gene BT (which codes for toxin production from the bacterium Bacillus thuringiensis) that is generally safe for human consumption [8]. Some genetically modified crops demonstrate insect resistance and hence alleviate pest control inputs and energy consumption. Moreover, they are environmentally friendly because farmers do not have to apply large amounts of pesticides and consequently involve less spraying to the surroundings which would in turn reduce the amount of harmful cancer causing mycotoxins in the environment Others argue that insect resistance will be only temporary because the pest would develop resistance at a later stage thus necessitating the use of even more pesticides. Indeed, this has already happened with GM cotton [1-7]. Crops can successfully reduce maturation time and improved resistance to different diseases along with its ability to increase stress tolerance. Some believe that GMF is the solution of world hunger because it increases food security every day and guarantees adequate nutrition while others argue that there is enough food around the globe for each person and that the problem which lies in food distribution will not simply be solved by GMF [9-11].

\section{Conclusion}

Rapid progress in the application of biotechnological methodologies and development of genetically modified food has significantly increased public concern about food safety in recent years. The release of GMFs into the environment and its enormous marketing has resulted in the dissemination of public debate in many parts of the world. This debate is likely to continue, probably in the broader context of other uses of biotechnology and their consequences for human societies. Even though the issues under dispute like safety, 
cost and benefits are usually very similar, the outcome differs from one country to another. Importantly however, on vital issues such as labeling and traceability of GMFs as a way to address consumer concerns, there is no consensus to date. Transcriptomics, proteomics and other related methods are not ready yet for routine use in the laboratory to test for GMF toxicity, however until clear evidence is provided all concerns can be considered relative and dealt with transparency and objectivity to reveal both the good and evil side of biotechnology.

\section{References}

1. Herbert M (2002) What is Genetically Modified Food :And Why Should You Care?. Research Foundation for Science, Technology and Ecology: Harvard Medical School.

2. Noussair C, Robin S (2002) Do Consumers Not Care About Biotech Foods or Do They Just Not Read The Label?. Economics Letter 72 : 47-53.

3. World Health Organization (2013) Fact sheet: Genetically Modified Food (GMF). WHO: Division of Healthy Environments and Sustainable Development, Food Safety Unit.

4. Huffman WE (2004) Production, identity preservation, and labeling in a marketplace with genetically modified and non-genetically modified foods. Plant Physiol 134: 3-10.
5. Hammer W (2006) Foods Derived from Genetically modified Organisms and Detection Methods. Genève, Swiss: close collaboration with the Swiss Federal Office of Public Health.

6. Pusztai A , Bardocz S (2003) Genetically modified foods: Potential Human Health Effects. Cambridge: CABI publishing.

7. Mims C (2004) Medical Microbiology: Third Edition. New York: ELSEVIER MOSBY.

8. Huffman W, Shogren J (2003) Consumer Willingness to pay for Genetically Modified Food Labels in a Market with Diverse Information: Evidence from Experimental Auctions. Journal of Agriculture and Resource Economics 28 : 481-502.

9. Gruere P (2003) International Approaches to the Labeling of Genetically ModifiedFoods. Department of Agriculture and Resource Economics, Davis: University of California.

10. Gruère P (2006) International Trade Regulations of GM Food: What Policies for Developing Countries?. Program for Biosafety Systems: International Food Policy Research Institute.

11. Gruere P (2006) An Analysis of Trade Related International Regulations of Genetically Modified Food and their Effects on Developing Countries. International Food Policy Research Institute: Environment and Production Technology Division. 